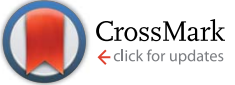

Cite this: RSC Adv., 2016, 6, 7455

\title{
Effect of shape and bending modulus on the properties of nematic lyotropic liquid crystals
}

Received 13th November 2015 Accepted 4th January 2016

DOI: $10.1039 / c 5 r a 24019 k$

www.rsc.org/advances

\author{
A. R. Ruiz-Fernández, ${ }^{\text {ab }}$ J. J. López-Cascales, ${ }^{\text {tb }}$ J. J. Giner-Casares, ${ }^{d}$ R. Araya- \\ Maturana, ${ }^{e}$ F. G. Díaz-Baños, ${ }^{c}$ D. Muñoz-Gacitúa ${ }^{a}$ and B. E. Weiss-López ${ }^{\star a}$
}

Synovial liquid is a natural lubricant of articular joints, such as shoulders, knees and hips. Thus, the development of biocompatible lubricants that can be employed in medical prosthesis for artificial implants, and eventually in certain therapies against osteo-arthritis or rheumatoid-arthritis diseases, is of an undoubted importance. Lyotropic liquid crystals have been used in the synthesis of silica and metals containing regularly oriented nanoporous, to obtain oriented carbon nanotube materials, in several pharmaceutical applications, as membrane mimetics and in lubrication applications. In this context, a new nematic lyotropic liquid crystal has been developed based on tetradecyltrimethyl ammonium chloride (TTAC), decanol (DeOH), a natural mixture of lipids (PL) extracted from soybean and sodium chloride $(\mathrm{NaCl})$, all dissolved in water. A $30 \% \mathrm{w} / \mathrm{w}$ increase in TTAC content respect a certain composition of reference produces a more than 24-fold increase in the macroscopic viscosity of the solution. To understand the molecular principles that explain this behavior, several experimental and theoretical studies have been carried out. In this regard, ${ }^{2} \mathrm{H}-\mathrm{NMR}$ quadrupole splittings of fully deuterated sodium dodecyl sulphate (SDS- $\mathrm{d}_{25}$ ) introduced as molecular probe, transmission electron microscopy (TEM), freeze fracture transmission electron microscopy (FF-TEM), cryogenic transmission electron microscopy (Cryo-TEM) and polarized light microscopy (PLM) were obtained. In addition, hydrodynamic studies determined how the enhancement in viscosity requires a change in the shape of the aggregate, from circular to elliptic. Finally, molecular dynamics simulations allowed to estimate how variations in the bending modulus, $k^{\mathrm{b}}$, can explain the observed tendency of the quadrupole splittings, on the basis of a modification in the flexibility of the aggregate.

\section{Introduction}

Synovial liquid plays a crucial role as a natural lubricant and shock absorption of articular joins such as hips, shoulders and knees, where an optimal lubrication is required for the proper joint mobility and a reduction in cartilages degradation..$^{1,2}$ In this regard, a deficiency in hyaluronic acid as a consequence of degradation in chronic diseases, such as osteo-arthritis and rheumatoid-arthritis, reduces the lubrication ability of synovial liquid, increasing the wearing of cartilages in knees, shoulders and hips. ${ }^{1-5}$ Furthermore, the sphingolipid and cholesterol composition of synovial liquid have been investigated with the

\footnotetext{
${ }^{a}$ Uni. de Chile, Fac. de Ciencias, Departamento de Quimica, Casilla 653, Santiago, Chile.E-mail: bweiss@uchile.cl

${ }^{b}$ Uni. Politécnica de Cartagena, Grupo de Bioinformática y Macromoléculas (BioMac), Campus de Alfonso XIII, Aulario II, 30203 Cartagena, Murcia, Spain. E-mail: javier. lopez@upct.es

'Uni. de Murcia, Fac. de Química, Dep. de Química Física, Campus de Espinardo, 30100 Espinardo, Murcia, Spain

${ }^{d}$ CIC biomaGUNE, Biofunctional Nanomaterials - Laboratory 6 Parque tecnológico de San Sebastián, Ed. Miramón 182, Guipúzcoa, 20009 Donostia - San Sebastián, Spain ${ }^{e}$ Uni. de Talca, Inst. de Quimica de Recursos Naturales, Casilla 747, Talca, Chile
}

goal of deciphering the role of these lipids in the lubrication of natural joints. ${ }^{1,2}$

By increasing the concentration of amphiphiles in aqueous solution above certain value, known as critical micellar concentration (CMC), it is possible to observe the formation of a variety of different molecular organizations or phases. The main forces that drive these aggregation processes are of hydrophobic and electrostatic nature. These materials have been used in the synthesis of silica and metals containing regularly oriented nanoporous, ${ }^{6-11}$ to obtain oriented carbon nanotube materials, ${ }^{12-14}$ as carriers in pharmaceutical formulations ${ }^{15-17}$ as membrane mimetics ${ }^{18-20}$ and in lubrication applications. ${ }^{21}$

In this context, Kupchinov et $a .^{4}$ determined that synovial liquid contains about $2 \%$ nematic liquid crystals, to which important lubrication effects have been associated. The particular rheological properties and bio-compatibility of nematic lyotropic liquid crystals made of natural phospholipids makes them excellent candidates to be used as lubricants in joints of medical prosthesis for artificial implants and in potential therapeutic applications. Nematic lyotropic liquid crystals of finite size can be spontaneously generated in the laboratory 
when amphiphiles are dissolved in water in the presence of aliphatic alcohols and salt. Thus, at least two types of aggregates can be spontaneously generated: calamitic $\left(\mathrm{N}_{\mathrm{C}}\right)$ derived from hexagonal phases with prolate average symmetry, and discotic $\left(\mathrm{N}_{\mathrm{D}}\right)$, derived from the lamellar phase with oblate average symmetry. ${ }^{22-24}$ Searching for the development of an artificial and bio-compatible nematic liquid crystal that can be used as lubricant in prosthetic joints for medical applications, and eventually as substitute of synovial liquid in certain therapeutic applications, ${ }^{25}$ different liquid crystals have been prepared from the mixture of tetradecyltrimethyl ammonium chloride (TTAC), decanol (DeOH), a natural mixture of phospholipids (PL) extracted from soybean and sodium chloride $(\mathrm{NaCl})$, all in aqueous solution. Few $\mathrm{mg}$ of fully deuterated sodium dodecylsulphate (SDS- $\mathrm{d}_{25}$ ) were added as a probe to test for the mobility of the aliphatic chains towards the interior of the hydrophobic core. The main advantage of using these liquid crystals as lubricant is that it is possible to modulate its lubricating power. In effect, an increase of $30 \% \mathrm{w} / \mathrm{w}$ in TTAC content, produces a more than 24 -fold increase in the solution viscosity. Thus, small variations in TTAC composition allows to modulate the lubricating properties of the solution within a wide range of values.

In this article, we focused our efforts on explaining how the kinematic viscosity of the liquid crystal is related to the size, shape and flexibility of the molecular aggregate. To characterize the structure of the aggregates, different experimental and theoretical studies have been carried out. Thus, to observe the order degree of the molecular packing along the aliphatic chains of the aggregate, $\mathrm{a}^{2} \mathrm{H}$-NMR study was performed in the presence of SDS- $\mathrm{d}_{25}$, used as molecular probe. The kinematic viscosity of the solutions were measured at $298 \mathrm{~K}$ in all the studied concentration range. To explore the shape and size of the molecular aggregates, Transmission Electron Microscopy (TEM), freeze fracture transmission electron microscopy (FFTEM) and Cryo-transmission electron microscopy (Cryo-TEM) images of selected samples were obtained. To assist with the characterization of the phases, polarized light microscopy (PLM) textures were also observed. With the aim of obtaining detailed insights into the properties of these liquid crystals, several molecular dynamics (MD) simulations were carried out with atomic detail. Finally, on the basis that our MD simulations are not capable to provide information related with the molecular rearrangements that should take place to explain the increase in viscosity with the increase in TTAC, a hydrodynamic modeling study was performed to explain the experimental observations.

\section{Materials and methods}

\subsection{Mesophase preparation}

PL from soybean, SDS- $\mathrm{d}_{25}$, TTAC and DeOH were purchased from Aldrich and used as received. Water of HPLC grade and $\mathrm{NaCl}$ at the highest purity available were purchased from Merck and used as received. The liquid crystal solutions were prepared by initially dissolving $0.2140 \mathrm{~g}$ of TTAC, $0.1165 \mathrm{~g}$ of PL, $0.012 \mathrm{~g}$ of SDS- $\mathrm{d}_{25}, 0.0912 \mathrm{~g}$ of $\mathrm{NaCl}$ and $58 \mu \mathrm{l}$ of DeOH in $1 \mathrm{ml}$ of $\mathrm{H}_{2} \mathrm{O}$.
Furthermore, the TTAC concentration was increased up to a total of $0.2930 \mathrm{~g}$ of TTAC, maintaining the other components constant. This TTAC content corresponds the range of TTAC concentrations in which the liquid crystal generated exists. All mesophases were allowed to equilibrate at least 48 hours at 300 $\mathrm{K}$ before any measurements were made. These mesophases displayed thermal stability up to $315 \mathrm{~K}$.

\section{$2.2{ }^{2} \mathrm{H}-\mathrm{NMR}$ spectra}

All NMR spectra were obtained at $300 \mathrm{~K}$ in a Bruker Avance 400 NMR spectrometer, located at the Universidad de Santiago de Chile, using a broadband probe tuned to $61.425 \mathrm{MHz}$. The ${ }^{2} \mathrm{H}$ $90^{\circ}$ pulse was $19 \mu \mathrm{s}$ long and more than 1000 transients from a spectral window of $40 \mathrm{kHz}$ were accumulated in $32 \mathrm{kB}$ files.

\subsection{Polarized light microscopy (PLM) and viscosity $(\eta)$}

A Motic series B microscope equipped with crossed polarizers was employed to observe the textures. The samples were placed in a concave slide with a depth of $1 \mathrm{~mm}$ and were allowed to orient in a $2.35 \mathrm{~T}$ magnet for $15 \mathrm{~min}$; longer periods in the field had no effects on the textures. The photos were taken at $300 \mathrm{~K}$ with a 20 -fold magnification, from the center of the concavity, avoiding variations in the thickness of the sample due to the curvature of the slide.

Kinematic viscosities of the liquid crystals, $\eta$, were measured using a standard Ubbelohde viscometer at $298 \mathrm{~K}$ and 1 atmosphere. Within the range of TTAC content, a noticeable change in the slope of the plot viscosity $v s$. TTAC concentration was observed. This is attributed to a change of phase and is corroborated with the PLM textures modification, observed at the same TTAC concentration. In this context, an special interest was paid on the following three experimental samples, which correspond to different phases of the synthesized liquid crystals:

Sample 1: this sample was prepared with $0.2250 \mathrm{~g}$ of TTAC, $0.1165 \mathrm{~g}$ of PL, $0.012 \mathrm{~g}$ of SDS- $\mathrm{d}_{25}, 0.0912 \mathrm{~g}$ of $\mathrm{NaCl}$ and $58 \mu \mathrm{l}$ of DeOH in $1 \mathrm{ml}$ of $\mathrm{H}_{2} \mathrm{O}$.

Sample 2: this sample was prepared with $0.2930 \mathrm{~g}$ of TTAC, $0.1165 \mathrm{~g}$ of PL, $0.012 \mathrm{~g}$ of SDS- $\mathrm{d}_{25}, 0.0912 \mathrm{~g}$ of NaCl and $58 \mu \mathrm{l}$ of DeOH in $1 \mathrm{ml}$ of $\mathrm{H}_{2} \mathrm{O}$. In summary, this phase was prepared starting from sample 1 in which the TTAC content was increased by $30 \% \mathrm{w} / \mathrm{w}$ and the rest of the components were maintained constant.

Sample 3: this sample was prepared with $0.2250 \mathrm{~g}$ of TTAC, $0.1165 \mathrm{~g}$ of PL, $0.012 \mathrm{~g}$ of SDS- $\mathrm{d}_{25}, 0.1048 \mathrm{~g}$ of $\mathrm{NaCl}$ and $58 \mu \mathrm{l}$ of $\mathrm{DeOH}$ in $1 \mathrm{ml}$ of $\mathrm{H}_{2} \mathrm{O}$. We note that sample 3 and 1 are of equal composition except that the $\mathrm{NaCl}$ content was increased by $15 \% \mathrm{w} / \mathrm{w}$.

\subsection{Transmission electron microscopy (TEM)}

TEM images were collected with a JEOL JEM-1400PLUS instrument operating at $120 \mathrm{kV}$, using carbon coated 400 square mesh copper grids. Samples were drop casted onto the TEM grids. 


\subsection{Freeze fracture transmission electron microscopy (FF- TEM)}

FF-TEM samples were prepared according to a previously described methodology. ${ }^{26}$ The solution was deposited between two copper platelets using a 400-mesh gold grid spacer. The samples were frozen by immersion in liquid propane, at $-189^{\circ} \mathrm{C}$, and fractured at $-150{ }^{\circ} \mathrm{C}$ and $10^{-8}$ mbar in a BAF 060 freeze-etching system (Leica Microsystems, Vienna). The replicas were obtained by unidirectional shadowing at $45^{\circ}$ with $2 \mathrm{~nm}$ of $\mathrm{Pt} / \mathrm{C}$ and at $90^{\circ}$ with $20 \mathrm{~nm}$ of C; they were floated on distilled water during $5 \mathrm{~min}$ and observed at $120 \mathrm{kV}$ in a Tecnai Spirit microscope (FEI Company, Eindhoven, Netherlands).

\subsection{Cryo-transmission electron microscopy (Cryo-TEM)}

Dipping and withdrawing a bare glow-discharged holey carbon grid from the solution a thin film was formed. The grid was blotted against filter paper, leaving thin sample films spanning the grid holes. These films were vitrified from room temperature by dipping into boiling ethane, using a Vitrobot (FEI Company, Eindhoven, Netherlands). The sample was kept at $100 \%$ humidity before freezing. Then it was transferred to a Tecnai F20 microscope (FEI Company, Eindhoven, Netherlands) using a Gatan cryoholder (Gatan, Pleasanton, CA). Images were taken at $200 \mathrm{kV}$, below $-170{ }^{\circ} \mathrm{C}$, using low-dose imaging conditions and an Eagle $4 \mathrm{k} \times 4 \mathrm{k}$ CCD camera (FEI Company, Eindhoven, Netherlands).

\subsection{Setting up the computational boxes}

To explore the effects of TTAC concentration and ionic strength on the properties of these liquid crystals, we have performed 3 simulations, representing sample 1,2 and 3 as bilayer fragments. Consequently, three computational boxes were generated, with periodic boundary conditions in all directions of space.

(1) System 1: this system was constituted by 392 tetradecyltrimethyl ammonium ion $\left(\mathrm{TTA}^{+}\right)+72$ phosphatidylcholine (DOPC) + 32 phosphatidylethanolamine (POPE) + 200DeOH + 32 dodecylsulphate ion $\left(\mathrm{DS}^{-}\right)+32$ cis-10-octadecanoic acid (AOL) + 32 sodium ions $\left(\mathrm{Na}^{+}\right)+392$ chloride ions $\left(\mathrm{Cl}^{-}\right)$. We must mention in this point, that $\mathrm{Na}^{+}$and $\mathrm{Cl}^{-}$are not balanced in the system, because they were introduced only for maintaining the electroneutrality of the system $\left(392 \mathrm{Cl}^{-}\right.$are required to balance the $392 \mathrm{TTA}^{+}$and $32 \mathrm{Na}^{+}$are required to balance the $32 \mathrm{DS}^{-}$). The dimensions of the original computational box were 10.3, 10.3 and $9.5 \mathrm{~nm}$ for the $x, y$, and $z$ axis, respectively, and the molecular ratios among $\mathrm{TTA}^{+}$, DOPC, POPE, DeOH and DS were close to the experimental values. This computational box is a representation of the experimental sample 1 (see Section 2.3), corresponding to the liquid crystal with $0.2250 \mathrm{~g}$ of TTAC.

(2) System 2: in this system, the number of TTAC was increased by $30 \%$ with respect to system 1 . Thus, the total number of molecules that constituted this system resulted as follows: $512 \mathrm{TTA}^{+}+72 \mathrm{DOPC}+32 \mathrm{POPE}+200 \mathrm{DeOH}+32 \mathrm{DS}^{-}+$ $32 \mathrm{AOL}+32 \mathrm{Na}^{+}+512 \mathrm{Cl}^{-}$. The starting dimensions of the computational box were $12.2,12.2$ and $10.6 \mathrm{~nm}$, for the $x, y$ and $z$ axis, respectively. In this case, this computational box is a representation of the experimental sample 2 (see Section 2.3), which corresponds to the liquid crystal with $0.2930 \mathrm{~g}$ of TTAC.

(3) System 3: in this case, system 1 was simulated including $\mathrm{NaCl}$ at $1 \mathrm{M}$ concentration. The dimensions of the starting computational box were $10.4,10.4$ and $9.6 \mathrm{~nm}$, for the $x, y$ and $z$ axis, respectively. In this case, this computational box is a representation of the experimental sample 3 (see Section 2.3), which corresponds to the liquid crystal with $0.2250 \mathrm{~g}$ of TTAC (sample 1) with an increase in the ionic strength.

In all three system described above, the species were symmetrically distributed between the two leaflets that constitute the bilayer. Fig. 1 shows a snapshot of system 1 and system 2 after 100 ns of trajectory.

\subsection{MD simulation parameters}

Once all three systems mentioned above were generated, the steepest descent minimization method was applied to remove the excess of energy associated with the overlapping between adjacent atoms. Once the energy achieved a minimum, $200 \mathrm{~ns}$ of trajectory length were simulated for each one of the systems previously described. GROMACS package 4.5.3 was used to carry out the molecular dynamics simulations. In this regard, an integration time step of 4 fs was employed. van der Waals interactions were simulated using the Lennard Jones potential (LJ), and long range electrostatic interactions were calculated using the Particle Mesh Ewald (PME) method. ${ }^{27,28}$ In both cases, for the LJ and the electrostatic interactions, a cut-off of $1.2 \mathrm{~nm}$ was employed. The bond lengths were restrained using the LINCS algorithm. ${ }^{29}$ All simulations were carried out in a NPT ensemble at $300 \mathrm{~K}$ and $1 \mathrm{~atm}$, coupled to weak temperature and pressure baths algorithms, ${ }^{30}$ with time constants of 0.1 ps and 1 ps for temperature and pressure, respectively. The RyckaertBellemans potential ${ }^{31}$ was used in all torsions along the
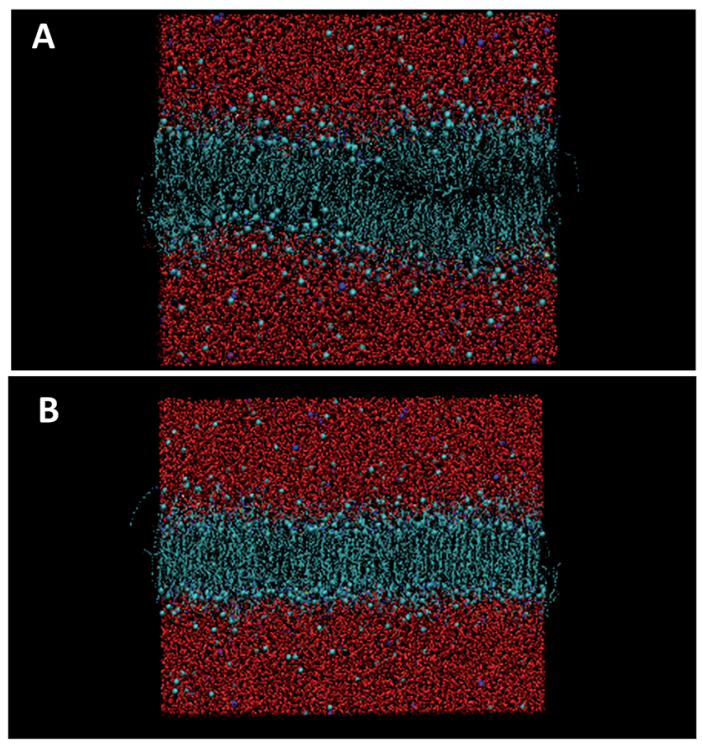

Fig. 1 Snapshots of the system 1 (A) and system 2 (B). For further information, see the text. 
Table 1 CNDO atomic charges of all molecules involved in this study, according to the atom numeration of Fig. 2

\begin{tabular}{|c|c|c|c|c|c|c|}
\hline Atom number & $\mathrm{TTA}^{+}$ & DOPC & POPE & $\mathrm{DeOH}$ & $\mathrm{DS}^{-}$ & $\mathrm{AOL}$ \\
\hline 1 & 0.400 & 0.40 & 0.645 & 0.426 & -0.404 & 0.24 \\
\hline 2 & 0.400 & 0.40 & 0.148 & -0.722 & -0.404 & -0.24 \\
\hline 3 & 0.400 & 0.40 & 0.005 & 0.329 & -0.404 & 0.41 \\
\hline 4 & -0.500 & -0.50 & -0.383 & -0.033 & 0.418 & -0.304 \\
\hline 5 & 0.300 & 0.30 & 0.447 & 0.0 & -0.286 & -0.106 \\
\hline 6 & 0.0 & 0.40 & -0.256 & 0.0 & 0.108 & 0.0 \\
\hline 7 & 0.0 & -0.80 & -0.259 & 0.0 & -0.028 & 0.0 \\
\hline 8 & 0.0 & 1.70 & -0.347 & 0.0 & 0.0 & 0.0 \\
\hline 9 & 0.0 & -0.80 & 0.060 & 0.0 & 0.0 & 0.0 \\
\hline 10 & 0.0 & -0.80 & 0.109 & 0.0 & 0.0 & 0.0 \\
\hline 11 & 0.0 & -0.70 & -0.232 & 0.0 & 0.0 & 0.0 \\
\hline 12 & 0.0 & 0.40 & 0.380 & 0.0 & 0.0 & 0.0 \\
\hline 13 & 0.0 & 0.30 & -0.344 & - & 0.0 & 0.0 \\
\hline 14 & 0.0 & -0.70 & 0.027 & - & 0.0 & 0.0 \\
\hline 15 & 0.0 & 0.070 & 0.0 & - & 0.0 & 0.0 \\
\hline 16 & 0.0 & -0.70 & 0.0 & - & 0.0 & 0.0 \\
\hline 17 & 0.0 & 0.0 & 0.0 & - & 0.0 & 0.0 \\
\hline $18-21$ & - & 0.0 & 0.0 & - & - & 0.0 \\
\hline $22-30$ & - & 0.0 & 0.0 & - & - & 0.0 \\
\hline 31 & - & 0.0 & 0.121 & - & - & - \\
\hline 32 & - & 0.0 & -0.233 & - & - & - \\
\hline 33 & - & 0.0 & 0.426 & - & - & - \\
\hline 34 & - & 0.50 & -0.341 & - & - & - \\
\hline 35 & - & -0.70 & 0.027 & - & - & - \\
\hline 36 & - & 0.8 & 0.0 & - & - & - \\
\hline 37 & - & -0.6 & 0.0 & - & - & - \\
\hline $38-53$ & - & 0.0 & 0.0 & - & - & - \\
\hline Total charge & 1.0 & 0.0 & 0.0 & 0.0 & -1.0 & 0.0 \\
\hline
\end{tabular}

aliphatic chains, for a better reproduction of cis-trans transitions.

The Lennard-Jones parameters were the same than those specified in a previous work, ${ }^{32}$ along with the standard GROMOS 54a7 force field. ${ }^{33}$ The atomic charges distribution of TTA $^{+}$, $\mathrm{DS}^{-}$, DeOH, DOPC, POPE and AOL were calculated using the semi-empirical Complete Neglect of Differential Overlap (CNDO) method. ${ }^{34}$ Table 1 shows the atomic charge distributions (in e units), considering the atom numeration of Fig. 2. Such as argued in a previous work, ${ }^{32}$ the charge distributions of all species with net charge were halved, to consider the absence of polarizability in all molecules involved and the constancy of

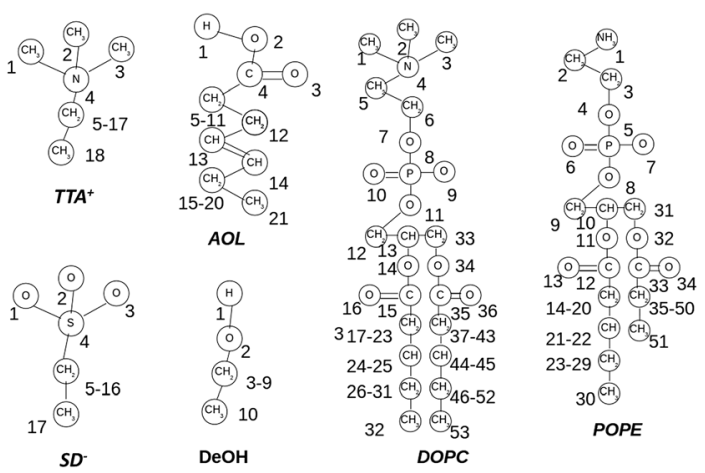

Fig. 2 Atoms numeration of all molecules involved in the simulations. the dielectric medium. ${ }^{32,35-40}$ Simple Point Charge (SPC) water model $^{41}$ was employed in all simulations.

\section{Results and discussion}

\subsection{Characterization of the molecular aggregates}

Fig. 3 shows the ${ }^{2} \mathrm{H}$-NMR spectra of SDS- $\mathrm{d}_{25}$ dissolved in sample 1 (A) and in sample 2 (B). The presence and magnitude of the quadrupole splittings along with the line shape of both spectra, strongly suggests that the aggregates correspond to bilayer structured micelles with nematic order. ${ }^{42-46}$ It is important to emphasize that these aggregates can not be spherical micelles since they can not generate ${ }^{2} \mathrm{H}-\mathrm{NMR}$ quadrupole splittings.

Table 3 shows the experimentally measured quadrupole splittings of SDS- $\mathrm{d}_{25}$ in samples 1, 2 and 3 (sample compositions were described in Section 2.3). A significant decrease in the splittings is observed when increasing the TTAC content, however both spectra are very similar. Accepting that in the range of studied concentrations the average orientation of the aggregate in the magnetic field is not modified, the value of the observed ${ }^{2} \mathrm{H}$-NMR quadrupole splitting of a given $\mathrm{C}-\mathrm{D}$ bond in a deuterated probe, such as SDS- $\mathrm{d}_{25}$, is a measurement of the alignment of that $\mathrm{C}-\mathrm{D}$ bond with the spectrometer magnetic field; in other words it is representative of the mobility of the C-D bond. Therefore we can test for modifications in the dynamics of the aliphatic chains at different depths towards the interior of the hydrophobic core. A significant decrease in the general alignment is observed when augmenting the TTAC content. In addition, both mesophases display a region, near the interface, that shows similar mobility; in particular, this region increases from $\mathrm{C} 1$ to $\mathrm{C} 5$ in sample 1 to $\mathrm{C} 1$ to $\mathrm{C} 7$ in sample 2.

Despite the similarities between the ${ }^{2} \mathrm{H}-\mathrm{NMR}$ spectra of sample 1 and sample 2, they present very different polarized light microscopy textures (see Fig. 3C and D). This observation evidences the existence of two different nematic mesophases, necessarily arising from structural changes in the aggregates. However, this change does not seems to affect the bilayer structure of the micelles, as evidenced from the ${ }^{2} \mathrm{H}-\mathrm{NMR}$ spectra. The oily streaks texture observed in Fig. 3C usually appears as a consequence of homeotropic order, generally observed in lamellar phases, however, sometimes it is displayed by nematic discotic lyotropic liquid crystals, such as in this case. The texture observed in Fig. 3D is the typical schlieren texture, usually observed in nematic mesophases. ${ }^{47}$ These textures can not arise from smectic phases since these phases display a ${ }^{2} \mathrm{H}$-NMR spectrum typical of a powder pattern; smectic phases do not orient in the spectrometer magnetic field. All discussed evidence strongly suggest that the structure of the aggregates should be associated to some type of bilayer structured micelles.

Fig. 4 shows Cryo-TEM (A) and FF-TEM (B) images of sample 1. Both figures corroborate the existence of individual aggregates of finite size, strongly suggesting the presence of a nematic phase. In particular, aggregates of about 2 to $5 \mathrm{~nm}$ can be observed from both figures, however clusters of aggregates can also be observed, which could be the origin of the oily streaks observed in PLM. In an effort to experimentally explore variations in the size of the aggregates with the modification in 
A)

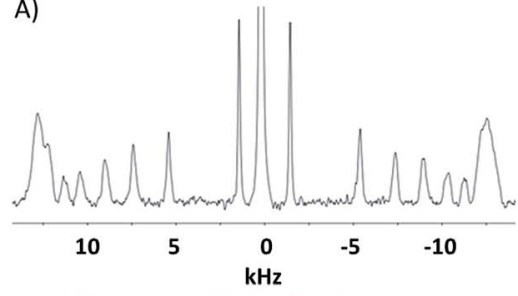

C)

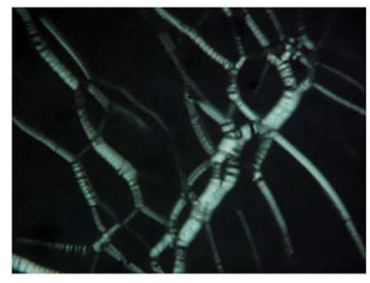

B)

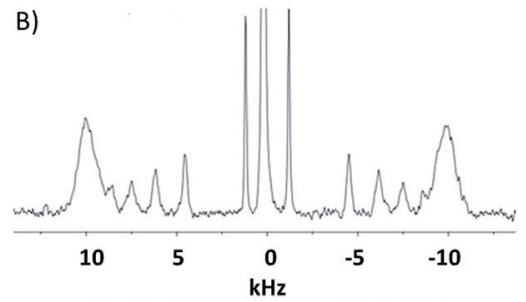

D)

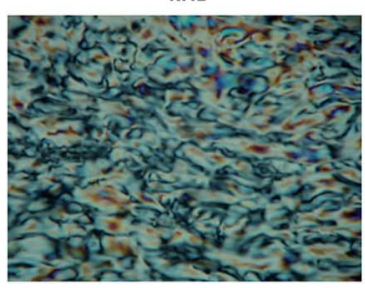

Fig. 3 (A) and (B) show the ${ }^{2} \mathrm{H}$-NMR spectrum of sample 1 and sample 2 (see Section 2.3) respectively. Quadrupolar splitting of SDS- $d_{25}$ can be measured directly from the spectra. (C) and (D) show polarized light microscopy textures observed in sample 1 and sample 2 respectively.
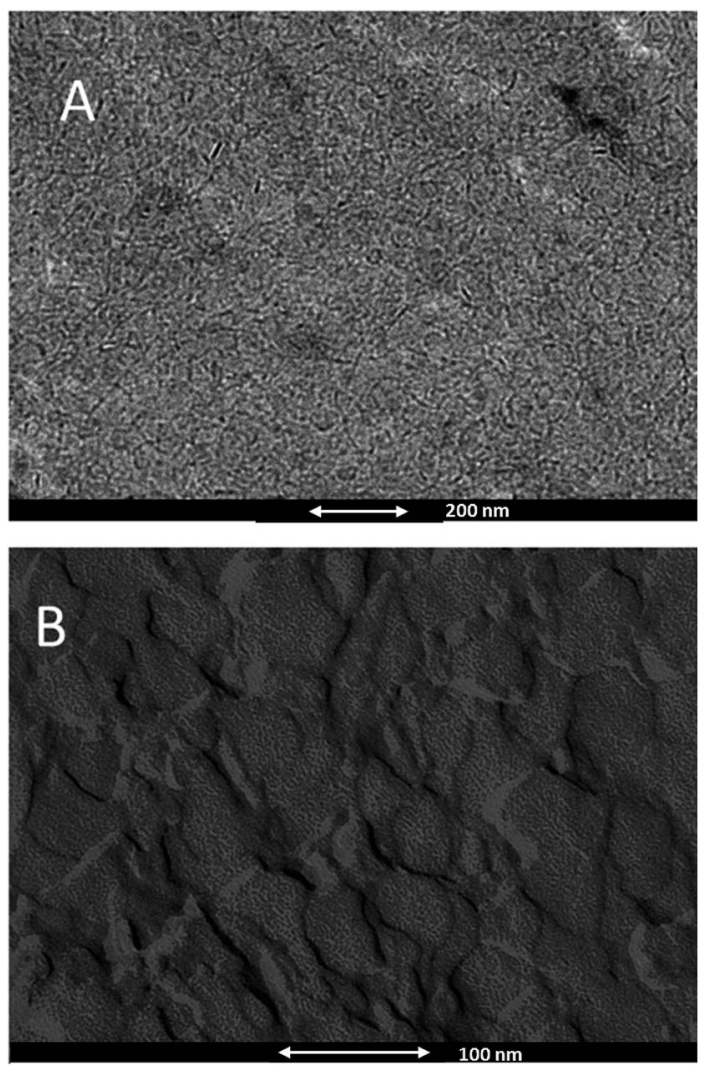

Fig. 4 (A) Cryo-TEM image of sample 1 and (B) FF-TEM image of sample 1. Sample compositions were described in Section 2.3.

TTAC content, TEM images of samples 1 and 2 were also obtained (Fig. 5). All TEM results, allow to discard the presence of lamellar or hexagonal smectic phases, usually surrounding the nematic region of the phase diagram. ${ }^{48} \mathrm{~A}$ simple comparison of the images in Fig. 5A and B show that aggregates of roughly 10-20 $\mathrm{nm}$ are observed in sample 2, large when compared with the image of sample 1, in which aggregates of scarcely 1-2 nm diameter can be observed. These experimental results are in excellent agreement with a hydrodynamic modeling calculation presented below.

\subsection{Aggregate bending modulus $\left(k^{b}\right)$}

The flexibility of the molecular aggregates that constitute the liquid crystal were estimated through the calculation of the bending modulus, $k^{\mathrm{b}}$, defined as: ${ }^{49}$

$$
k^{\mathrm{b}}=\frac{K_{\mathrm{A}} \xi^{2}}{24}
$$

here $\xi$ is called the effective bilayer thickness, defined by $\xi=d-$ 1 where $d$ is the distance between the maxima of the nitrogen atom distributions of $\mathrm{TTA}^{+}$head-groups in both leaflets of the bilayer. $K_{\mathrm{A}}$ represents the area compressibility modulus, which is defined as follows:

$$
K_{\mathrm{A}}=\frac{k_{\mathrm{B}} T A}{\sigma^{2}(A)}
$$

where, $A$ and $\sigma^{2}(A)$ correspond to the mean and mean-squared fluctuation of the interfacial area per $\mathrm{TTA}^{+}$, respectively. $k_{\mathrm{B}}$ is the Boltzmann constant and $T$ the absolute temperature. Table 3 shows the calculated bending modulus, $k^{\mathrm{b}}$, using MD simulations.

Unfortunately, no experimental values to validate the bending modulus listed in Table 3 were found. However the values obtained here are in reasonable agreement with bending modulus obtained for lipid bilayers of different composition, where values from 15 to $30 k_{\mathrm{B}} T$ have been measured using X-ray,
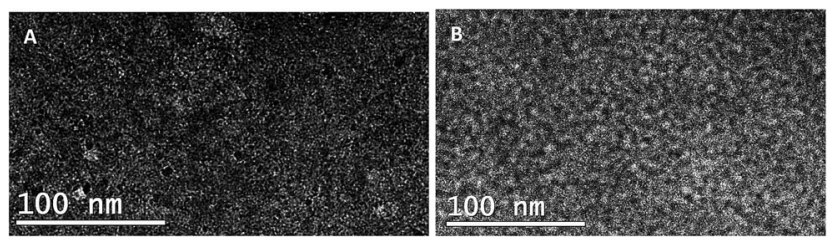

Fig. 5 TEM images corresponding to (A) sample 1 and (B) sample 2. Sample compositions were described in Section 2.3. 
pipette aspiration and neutron spin echo experiments, ${ }^{50-54}$ and from MD simulations. ${ }^{55}$

From the results shown in Table 3, we observe a reduction of more than $50 \%$ in the bending modulus of the bilayer with an increase of $30 \% \mathrm{w} / \mathrm{w}$ in TTAC content (or in other words, the flexibility of the aggregate becomes doubled with the increase in TTAC content). Furthermore, we observe how a moderate increase in ionic strength (which corresponds to system 3 of Section 2.7) produces less than $20 \%$ increment in the bending modulus, relative to system 1 . Therefore, as expected, an increase in ionic strength produces a moderate increase in the stiffness of the bilayer, in good agreement with the experimental results reported by Claessens et al. ${ }^{56}$ for a DOPG and DOPC bilayers at different ionic strength of the aqueous solution, and from molecular dynamics simulations of binary DPPC/DPPS bilayers in presence and absence of salt. ${ }^{55}$ Thus, this increase in bending modulus in system 3 is related with the fact that an increase in the ionic strength produces an additional stabilization of the interface, as expected from a counterion neutralization, that is reflected into an increase in the stiffness of the molecular aggregates.

From Table 3 we see how this increase in flexibility in sample 2 respect to sample 1 , arises mainly from a reduction in the thickness of the bilayer. This decrease is associated with an increase in the interdigitation degree of the $\mathrm{TTA}^{+}$hydrocarbon tails, since $K_{\mathrm{A}}$ and $A$ both remained almost invariable for all three studied cases.

\subsection{Viscosity}

Fig. 6 shows the kinematic viscosity of the liquid crystal as a function of TTAC content in the composition window in which the liquid crystal remains, outside of this windows it changes to a different isotropic phase. From Fig. 6 we appreciate a change in the slope of the viscosity with TTAC composition at a certain critical concentration of TTAC. Thus, values of $12.90 \pm 0.06$ and $310 \pm 4 \mathrm{mPa}$ s were obtained for samples 1 and 2 respectively (see Section 2.3 for their composition). For the case of sample 3 (in which $\mathrm{NaCl}$ increased $15 \% \mathrm{w} / \mathrm{w}$ respect to the sample 1), a viscosity of $10.28 \pm 0.06 \mathrm{mPa} s$ was measured, showing a moderate dependence on ionic strength. These results show an

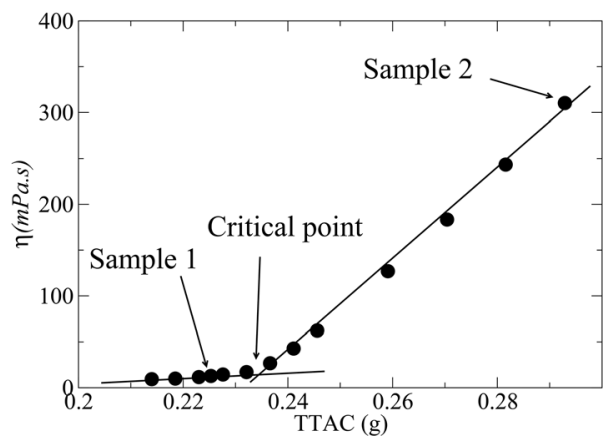

Fig. 6 Viscosity of the liquid crystal as a function of TTAC content (in grams) maintaining constant the rest of the components. increase in the solution viscosity with TTAC content, where an increase in TTAC of scarcely $30 \% \mathrm{w} / \mathrm{w}$ in sample 1 produces an increase of more than 24 times in viscosity of sample 2 respect to the measured in sample 1. From Fig. 6, the composition at which the slopes change corresponding to $0.234 \mathrm{~g}$ in TTAC, is coincident with the concentration at which changes in the PLM textures were observed, corroborating the occurrence of a phase transition.

This change in the viscosity slope will be associated to a change in size and shape of the molecular aggregates, such as it will be discussed in the section below.

3.3.1 Shape of molecular aggregates. A factor to consider in the explanation of the notorious increase in viscosity of the solution with TTAC content is related with the size and shape of the molecular aggregates. For this purpose, a deeper study, based on changes in size and shape of the aggregates, is presented.

The intrinsic viscosity, $[\eta]$, provides information related to the shape and flexibility of the species dissolved, such as synthetic polymers, macromolecules, nanoparticles, aggregates or colloids, ${ }^{57,58}$ where $[\eta]$ can be written as follows:

$$
[\eta]=\lim _{c \rightarrow 0} \frac{\eta_{\mathrm{sp}}}{c}=\lim _{c \rightarrow 0} \frac{\eta_{\mathrm{r}}-1}{c}
$$

being $\eta_{\mathrm{sp}}$ the specific viscosity of the micellar solution of concentration $c$ (in this case $c$ corresponds to the concentration of aggregates) and $\eta_{\mathrm{r}}$ is the relative viscosity calculated from the ratio, $\eta_{\mathrm{r}}=\eta / \eta_{0}$, where $\eta$ is the viscosity of the solution and $\eta_{0}$ the viscosity of pure solvent.

Solomon-Ciuta $^{59}$ eqn (4), provides a relation between the intrinsic viscosity $[\eta]$ of a solute and the viscosity $\eta$ of the solution:

$$
[\eta]=\frac{\left(2 \times\left(\eta_{\mathrm{sp}}-\ln \eta_{\mathrm{r}}\right)\right)^{1 / 2}}{c}
$$

where $[\eta]$ is the intrinsic viscosity (or viscosity at infinite dilution) described above.

Therefore, the Solomon-Ciuta equation can be rewritten as follows:

$$
[\eta] c=\left(2 \times\left(\eta_{\mathrm{sp}}-\ln \eta_{\mathrm{r}}\right)\right)^{1 / 2}
$$

The ratio between the intrinsic viscosities of two liquid crystals with different TTAC content can be calculated from eqn (6):

$$
\frac{[\eta]_{2} c_{2}}{[\eta]_{1} c_{1}}=\frac{\left(2 \times\left(\eta_{\mathrm{sp}, 2}-\ln \eta_{\mathrm{r}, 2}\right)\right)^{1 / 2}}{\left(2 \times\left(\eta_{\mathrm{sp}, 1}-\ln \eta_{\mathrm{r}, 1}\right)\right)^{1 / 2}}
$$

Assuming that $\eta_{0}$ is the viscosity of pure water at $298 \mathrm{~K}\left(\eta_{0}=\right.$ $1 \mathrm{mPa} \mathrm{s}$ ), and $\eta_{1}=12,9 \mathrm{mPa}$ and $\eta_{2}=310,2 \mathrm{mPa}$ for samples 1 and 2 respectively, eqn (6) can be reduced to the following expression:

$$
\frac{[\eta]_{2} c_{2}}{[\eta]_{1} c_{1}}=5.7
$$

From results of TEM microscopy, and considering that the size of the molecular aggregates for sample 2 is roughly 10 times 
bigger than for sample 1 and that TTAC composition increased scarcely a $30 \% \mathrm{w} / \mathrm{w}$ from sample 1 to sample 2 , we can assume that $c_{1} \simeq 10 c_{2}$, and then, eqn (7) can be re-written as follows,

$$
\frac{[\eta]_{2}}{[\eta]_{1}}=57.0
$$

In general, the intrinsic viscosity $[\eta]$ depends on the shape and size of the macromolecule or colloid in solution. At this point, the main goal is try to reproduce the ratio between intrinsic viscosities for liquid crystals of different TTAC content, using hydrodynamic calculations based on molecular aggregates of different size and shape.

Thus, the program HYDRO ${ }^{60}$ (available from http:// leonardo.inf.um.es/macromol), allows to calculate a dimensionless intrinsic viscosity. A model composed of two layers of 244 hard spheres each, forming a circular monoaxial structure, such as the one depicted in Fig. 7 was constructed. For this model, an intrinsic viscosity of $[\eta]_{1 \text {, circular }}^{*}=1.39$ (where the star means that we are working in dimensionless units) was calculated. To explain the increase in the viscosity of sample 2 , the intrinsic viscosity was calculated for two different shapes, circular and elliptic (with a ratio in its semi-axes $a / b=20$ ), with 2440 hard spheres that reproduce an increase in the surface area 10 times bigger than the corresponding to the circular shape in sample 1. From these calculations, $\mathrm{a}[\eta]_{2, \text { circular }}^{*}=6.25$ and $[\eta]_{2, \text { elliptic }}^{*}=75$ were obtained, which provide a $[\eta]_{2}^{*} /[\eta]_{1}^{*}$ ratio of $[\eta]_{2 \text {,circular }}^{*}$ $[\eta]_{1, \text { circular }}^{*}=4.5$ and $[\eta]_{2 \text {,elliptic }}^{*} /[\eta]_{1, \text { circular }}^{*}=54$, considering that the aggregates adopt an circular and elliptic shape in sample 2. From these calculations, we can assert that the increase in TTAC content, not only increases the size but also induce changes in the shape of the molecular aggregates that form the liquid crystal, going from a circular to an elliptic symmetry, and that this change in the shape in the molecular aggregates is the main responsible of the increase in the viscosity of the liquid crystal.

\section{$3.4 \quad{ }^{2}$ H-NMR study}

Table 2 shows the ${ }^{2} \mathrm{H}$-NMR quadrupole splittings $\left(\Delta \nu_{\mathrm{Q}}\right)$ of SDS$\mathrm{d}_{25}$ dissolved in samples 1,2 and 3 (samples details were described in Section 2.3).

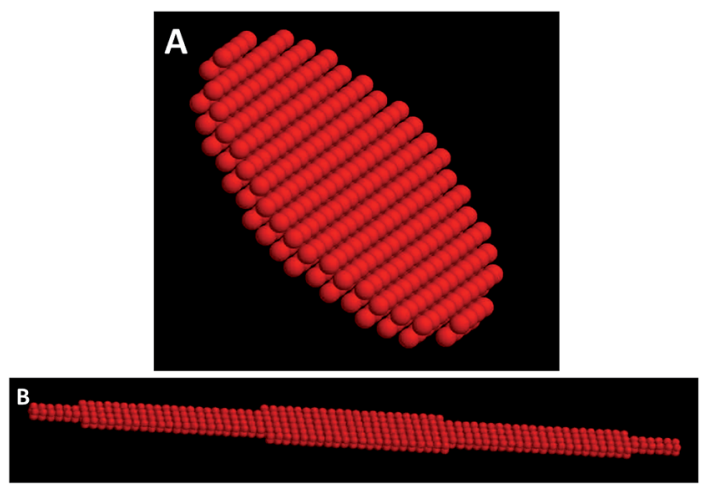

Fig. 7 Hydrodynamic models used in the estimation of [ $\eta]$. (A) Circle and (B) ellipse with $a / b=20$.
Table 2 Experimental values of ${ }^{2} \mathrm{H}$-NMR quadrupole splittings $\left(\Delta \nu_{\mathrm{Q}}\right)$ in $\mathrm{Hz}$. Sample compositions were described in Section 2.3

\begin{tabular}{llll}
\hline Carbon & $\begin{array}{l}\text { Sample } 1, \Delta \nu_{\mathrm{Q}} \\
(\mathrm{Hz})\end{array}$ & $\begin{array}{l}\text { Sample } 2, \Delta \nu_{\mathrm{Q}} \\
(\mathrm{Hz})\end{array}$ & $\begin{array}{l}\text { Sample } 3, \Delta \nu_{\mathrm{Q}} \\
(\mathrm{Hz})\end{array}$ \\
\hline 1 & 25371 & 20153 & 25232 \\
2 & 25371 & 20153 & 25232 \\
3 & 25371 & 20153 & 25232 \\
4 & 25371 & 20153 & 25232 \\
5 & 25371 & 20153 & 25232 \\
6 & 24783 & 20153 & 24489 \\
7 & 22734 & 20153 & 22564 \\
8 & 20750 & 17386 & 20663 \\
9 & 18016 & 15094 & 17710 \\
10 & 14815 & 12371 & 10792 \\
11 & 10852 & 9046 & 2854 \\
12 & 2889 & 2383 & \\
\hline
\end{tabular}

Table 2 shows how the quadrupole splittings of SDS- $\mathrm{d}_{25}$ significantly decreased with the increase in TTAC content. This indicates a significant decrease in the alignment of the aliphatic chains with the magnetic field, which could have different origins, including electrostatic repulsions among the ammonium head groups. On the other hand, an increase of $15 \% \mathrm{w} / \mathrm{w}$ in $\mathrm{NaCl}$ (sample 3) has no significant effect on the experimental splittings, showing that the integrity of aggregates does not depend markedly on ionic strength.

Values of $\Delta \nu_{\mathrm{Q}}$ obtained from ${ }^{2} \mathrm{H}-\mathrm{NMR}$ are related to deuterium order parameters, $S_{\mathrm{CD}}$, through the following expression:

$$
\Delta \nu_{\mathrm{Q}}=\frac{3 C\left\langle S_{\mathrm{CD}}\right\rangle}{2}
$$

$C$ is the quadrupole coupling constant ( $C=170 \mathrm{kHz}$ (ref. 61)), and $S_{\mathrm{CD}}$ is the order parameter of a given C-D bond. Order parameters can be extracted directly from simulations, using eqn (10).

$$
\left\langle S_{\mathrm{CD}}\right\rangle=\frac{\left\langle 3 \cos ^{2} \phi-1\right\rangle}{2}
$$

Here $\phi$ is the angle between the $\mathrm{C}-\mathrm{D}$ bond and the bilayer normal, and the average is carried out over the simulation time and the number of identical molecules in the computational box. However, and bearing in mind that hydrogens from methylene groups have not been explicitly considered, the order parameters corresponding to a given $\mathrm{C}-\mathrm{D}$ bond, can be

Table 3 Compressibility $\left(K_{A}\right)$ and bending modulus $\left(k^{b}\right)$ for the bilayers of different TTAC content, in the presence and absence of salt, estimated from simulations. A corresponds to the mean surface area per $\mathrm{TTA}^{+}$and $d$ is the average distance between nitrogen atoms of TTA ${ }^{+}$ head groups located in different leaflets of the bilayer. Systems 1, 2 and 3 were described in Section 2.7

\begin{tabular}{lcccc}
\hline System & $A\left(\mathrm{~nm}^{2}\right)$ & $d / \mathrm{nm}$ & $K_{\mathrm{A}} /\left(\mathrm{J} \mathrm{m}^{-2}\right)$ & $k^{\mathrm{b}} /\left(k_{\mathrm{B}} T\right)$ \\
\hline 1 & $0.5361 \pm 0.0007$ & 3.7 & $0.181 \pm 0.019$ & $12.9 \pm 1.4$ \\
2 & $0.574 \pm 0.003$ & 2.6 & $0.25 \pm 0.06$ & $6.1 \pm 1.4$ \\
3 & $0.537 \pm 0.007$ & 3.8 & $0.24 \pm 0.08$ & $16 \pm 3$
\end{tabular}


Table 4 Calculated values of ${ }^{2} \mathrm{H}$-NMR quadrupole splittings $\left(\Delta \nu_{\mathrm{Q}}, \mathrm{Hz}\right)$ from MD simulations. Systems 1, 2 and 3 were described in Section 2.7

\begin{tabular}{llll}
\hline Carbon & System $1, \Delta \nu_{\mathrm{Q}}(\mathrm{Hz})$ & System $2, \Delta \nu_{\mathrm{Q}}(\mathrm{Hz})$ & System $3, \Delta \nu_{\mathrm{Q}}(\mathrm{Hz})$ \\
\hline 1 & $25467 \pm 525$ & $24975 \pm 816$ & $24183 \pm 391$ \\
2 & $25639 \pm 663$ & $25596 \pm 844$ & $25483 \pm 589$ \\
3 & $26260 \pm 670$ & $26415 \pm 1110$ & $26721 \pm 559$ \\
4 & $26651 \pm 678$ & $26414 \pm 1254$ & $27390 \pm 583$ \\
5 & $26858 \pm 716$ & $26949 \pm 1360$ & $27600 \pm 622$ \\
6 & $26652 \pm 659$ & $27162 \pm 1354$ & $27566 \pm 692$ \\
7 & $25699 \pm 647$ & $27067 \pm 1439$ & $27299 \pm 717$ \\
8 & $25268 \pm 619$ & $26755 \pm 1390$ & $26864 \pm 690$ \\
9 & $23572 \pm 568$ & $26091 \pm 1388$ & $26019 \pm 711$ \\
10 & $20904 \pm 410$ & $24831 \pm 1236$ & $24489 \pm 586$ \\
11 & $16389 \pm 223$ & $22688 \pm 1020$ & $21038 \pm 557$
\end{tabular}

calculated employing a well described methodology, exhaustively explained elsewhere. ${ }^{32,38}$ Consequently, order parameter for $\mathrm{C}_{12}-\mathrm{D}$ bond can not be calculated. Table 4 shows the quadrupole splittings obtained from simulations, corresponding to the systems 1, 2 and 3 (described in Section 2.7) which correlate with the experimental samples 1, 2 and 3 described in Section 2.3.

An inspection to Tables 2 and 4 shows that a semiquantitative agreement between calculated splittings of SDS- $\mathrm{d}_{25}$ in system 1 and the experimental measured values in sample 1 , is obtained for the first few carbon atoms of the aliphatic chain, near the interface. However, a significant deviation emerged when we look at the last $\mathrm{C}-\mathrm{D}$ bonds of the chain. This discrepancy could arise either from the fact that our simulations corresponds to bilayer fragments, neglecting completely the curved rims which can be particularly important for small aggregates, or from molecules present as impurities in the PL mixture, which perturb the order of the hydrophobic core.

The observed ${ }^{2} \mathrm{H}$ quadrupole splitting along the SDS- $\mathrm{d}_{25}$ tail are not significantly altered by an increase in the solution ionic strength, as shown in Table 2 for samples 1 and 3. This behavior is reproduce by the simulation, as shown in Table 4 , for systems 1 and 3 respectively.

As mentioned before, comparing systems 1 and 2 with their corresponding experimental samples 1 and 2, a noticeable

Table 5 Calculated values of ${ }^{2} \mathrm{H}$-NMR quadrupole splittings $\left(\Delta \nu_{\mathrm{Q}}, \mathrm{Hz}\right)$ of system 2 (see Section 2.7 for different tilt angles with respect to the $Z$ axis: (a) $0^{\circ}$, (b) $10^{\circ}$ (c) $15^{\circ}$ and (d) $20^{\circ}$ )

\begin{tabular}{lllll}
\hline Carbon & $\left(\Delta \nu_{\mathrm{Q}}(\mathrm{Hz})\right)^{\mathrm{a}}$ & $\left(\Delta \nu_{\mathrm{Q}}(\mathrm{Hz})\right)^{\mathrm{b}}$ & $\left(\Delta \nu_{\mathrm{Q}}(\mathrm{Hz})\right)^{\mathrm{c}}$ & $\left(\Delta \nu_{\mathrm{Q}}(\mathrm{Hz})\right)^{\mathrm{d}}$ \\
\hline 1 & $24183 \pm 391$ & $21302 \pm 1211$ & $18440 \pm 1425$ & $14845 \pm 1718$ \\
2 & $25483 \pm 589$ & $22557 \pm 1354$ & $19603 \pm 1575$ & $15917 \pm 1882$ \\
3 & $26721 \pm 559$ & $23633 \pm 1268$ & $20525 \pm 1465$ & $16645 \pm 1752$ \\
4 & $27390 \pm 583$ & $24229 \pm 1253$ & $21048 \pm 1455$ & $17080 \pm 1755$ \\
5 & $27600 \pm 622$ & $24448 \pm 1237$ & $21235 \pm 1433$ & $17226 \pm 1726$ \\
6 & $27566 \pm 692$ & $24381 \pm 1231$ & $21178 \pm 1423$ & $17183 \pm 1720$ \\
7 & $27299 \pm 717$ & $24132 \pm 1246$ & $20955 \pm 1436$ & $16943 \pm 1724$ \\
8 & $26864 \pm 690$ & $23733 \pm 1199$ & $20599 \pm 1393$ & $16695 \pm 1681$ \\
9 & $26019 \pm 711$ & $22960 \pm 1183$ & $19910 \pm 1368$ & $16115 \pm 1646$ \\
10 & $24489 \pm 586$ & $21596 \pm 1123$ & $18720 \pm 1298$ & $15146 \pm 1561$ \\
11 & $21038 \pm 557$ & $18524 \pm 1063$ & $16036 \pm 1217$ & $12942 \pm 1443$
\end{tabular}

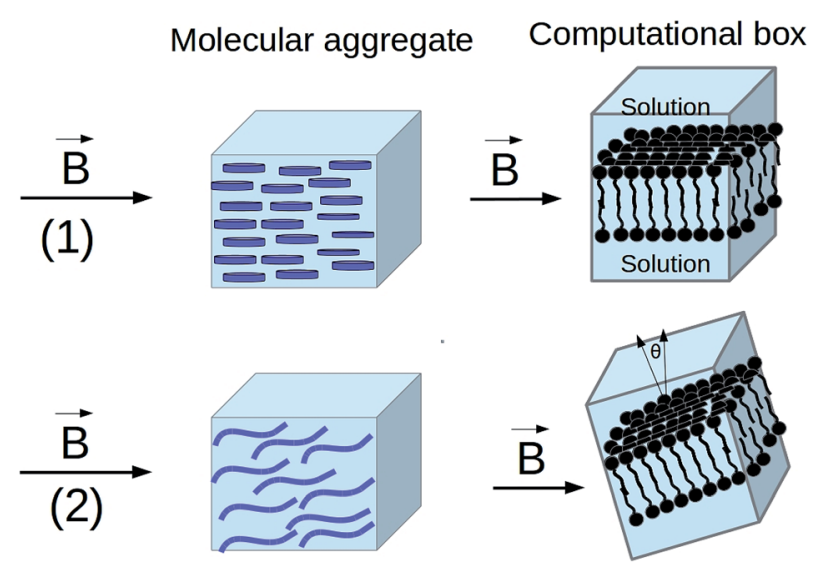

Fig. 8 Orientation of the aggregate with respect to the magnetic field direction. (1) Represents the orientation of stiff aggregates with oblate shape perfectly orientated in the magnetic field. (2) Represents distorted flexible aggregates with an elliptic shape, partially orientated respect to the magnetic field.

discrepancy between simulation and experiment arises. Experimentally it is observed how a moderate increase in TTAC content produces a reduction of about $19 \%$ in the quadrupole splitting for the first methylene groups of SDS- $\mathrm{d}_{25}$. This results clearly diverge from the results obtained from simulations, in which the same increase in TTAC content, produces no variation in the quadrupole splittings of the first C-D bonds, but a significant increment at the interior of the hydrophobic core.

This discrepancy between experiment and simulation can be explained on the basis of changes in the shape and flexibility of the aggregates when increasing the TTAC content, such as it was deduced in the previous Sections 3.2.1 and 3.2.2. Therefore, due to the increase in size and symmetry modifications of the aggregate, to a larger and elongated shape, there appear collective motions, similar to undulations observed in extended lamellar phases, which could increase the mobility of all $\mathrm{C}-\mathrm{D}$ bonds, decreasing the quadrupole splittings without affecting their average orientation. Thus, to explore the effect of undulations on $\Delta \nu_{\mathrm{Q}}$, values of $\Delta \nu_{\mathrm{Q}}$ have been recalculated considering four different tilt angles between the $\mathrm{C}-\mathrm{D}$ bonds and the magnetic field, and the results are displayed in Table 5. It is observed that an angle deviation of $\simeq 15^{\circ}$, such as it is represented in Fig. 8, is sufficient to reduce the values of $\Delta \nu_{\mathrm{Q}}$ close to the experimental values, as observed from Table 5. Therefore, undulations with oscillations of $15^{\circ}$ amplitude around the average orientation should be enough to explain the decrease in $\Delta \nu_{\mathrm{Q}}$. This result evidences how considerations of flexibility and size of the molecular aggregates are of crucial importance for the correct interpretation of the experimental data.

\section{Conclusions}

The use of molecular dynamics in conjunction with hydrodynamic calculations have proven to be very useful in the interpretation of the experimental results. 
From the change in the slope of the plot of kinematic viscosity $v s$. TTAC content, a phase transition process between different bilayer structured aggregates is evidenced. Additional experimental evidence to support this hypothesis arises from the observation of PLM texture modification, at the same concentration where the slope of viscosity $v s$. TTAC content is observed. Cryo-TEM, FF-TEM and room temperature TEM images point in the same direction.

Hydrodynamic calculations support a phase transition from disk shaped mono-axial aggregates to bigger elliptic shaped biaxial aggregates, explaining the viscosity increase. A decrease in bending modulus going from system 1 to system 2 is predicted by MD simulations. This increase in flexibility, which arises from the inter-digitation of $\mathrm{TTA}^{+}$hydrocarbon chains, also contributes to increase the viscosity.

Finally, the increase in size and flexibility of the aggregates allows collective motions to occur, which decreases the alignment of the C-D bonds with the magnetic field, decreasing the values of $\Delta \nu_{\mathrm{Q}}$. MD simulations indicates that oscillation amplitudes which deviates by $15^{\circ}$ the alignment with the field, should be sufficient to explain the observed splittings.

\section{Acknowledgements}

The authors are pleased to acknowledge financial support from Fondecyt-Chile (Grant No. 1150135), Universidad de Chile (VID Grant ENL-003 and Facultad de Ciencias). The authors also acknowledge the invaluable assistance from the staff and facilities at the Computing Center of the Universidad Politecnica de Cartagena and the National Laboratory for High Performance Computing (NLHPC), Universidad de Chile. ARRF acknowledges a doctoral fellowship from Conicyt. JJLC acknowledges the financial support from Fundación Séneca (19353/PI/14).

\section{References}

1 S. Sivan, A. Schroeder, G. Verberne, Y. Merkher, D. Diminsky, A. Priev, A. Maroudas, G. Halperin, D. Nitzan, I. Etsion and Y. Barenholz, Langmuir, 2009, 26, 1107-1116.

2 M. Knosinska, G. Liebisch, G. Lochnit, J. Wilhelm, H. Klein, U. Kaesser, G. Lasczkowski, M. Rickert, G. smitz and J. Steinmeyer, PLoS One, 2014, 9, e91769.

3 E. Waters, P. Spedding, A. Doherthy and R. Spedding, AsiaPac. J. Chem. Eng., 2009, 4, 80-89.

4 B. Kupchinov, S. Ermakov, V. Rodnenkov, S. Bobrysheva, E. Beloenko and V. Kestelman, Smart Mater. Struct., 1993, 2, 7-12.

5 C. Myant and P. Cann, J. Mech. Behav. Biomed. Mater., 2014, 34, 338-348.

6 A. Takai, Y. Yamauchi and K. Kuroda, J. Am. Chem. Soc., 2009, 132, 208-214.

7 S. Warren and U. Wiesner, Pure Appl. Chem., 2009, 81, 73-84. 8 X. Zhang, W. Lu, J. Dai, L. Bourgeois, J. Yao, H. Wang, J. Friend, D. Zhao and D. MacFarlane, Sci. Rep., 2014, 4, 7420.

9 S. Che, Z. Liu, T. Ohsuna, K. Sakamoto, O. Terasaki and T. Tatsumi, Nature, 2004, 429, 281-284.
10 H. Lin and C. Mou, Acc. Chem. Res., 2002, 35, 927-935.

11 S. Umadevi, H. Lee, V. Ganesh, X. Feng and T. Hegmann, Liq. Cryst., 2014, 41, 265-276.

12 G. Scalia, ChemPhysChem, 2010, 11, 333-340.

13 D. Vijayaraghavan, J. Mol. Liq., 2014, 199, 128-132.

14 M. Casavant, D. Walters, J. Schmidt and R. Smalley, J. Appl. Phys., 2003, 93, 2153-2156.

15 M. Cohen-Avrahami, A. Shames, M. Ottaviani, A. Aserin and

N. Garti, Colloids Surf., B, 2014, 122, 231-240.

16 C. Guo, J. Wang, F. Cao, R. Lee and G. Zhai, Drug Discovery Today, 2010, 15, 1032-1040.

17 M. Ki, J. Lim, J. Ko, S. Park, J. Kim, H. Cho, E. Park and D. Kim, J. Controlled Release, 2014, 185, 62-70.

18 V. Bahamonde-Padilla, J. López-Cascales, R. ArayaMaturana, M. Martínez-Cifuentes and B. Weiss-López, ChemPhysChem, 2014, 15, 1422-1431.

19 U. Dürr, M. Gildenberg and A. Ramamoorthy, Chem. Rev., 2012, 112, 6054-6074.

20 D. Warschawski, A. Arnold, M. Beaugrand, A. Gravel, É. Chartrand and I. Marcotte, Biochim. Biophys. Acta, Biomembr., 2011, 1808, 1957-1974.

21 L. Pinchuk, Y. Chernyakova and V. Goldade, J. Frict. Wear, 2008, 29, 224-233.

22 A. Nesrullajev, F. Tepehan and N. Kazanci, Mater. Chem. Phys., 2002, 73, 74-77.

23 R. Montecinos, H. Ahumada, R. Araya-Maturana, A. Olea and B. Weiss-López, J. Colloid Interface Sci., 2007, 316, 126-131.

24 A. Neto and S. Salinas, The Physics of Lyotropic Liquid Crystals: Phase Transitions and Structural Properties: Phase Transitions and Structural Properties, Oxford University Press, 2005.

25 J. Lagerwall and G. Scalia, Curr. Appl. Phys., 2012, 12, 13871412.

26 O. López, A. de la Maza, L. Coderch, C. López-Iglesias, E. Wehrli and J. Parra, FEBS Lett., 1998, 426, 314-318.

27 T. Darden, D. York and L. Pedersen, J. Chem. Phys., 1993, 98, 10089-10092.

28 U. Essmann, L. Perera, M. Berkowitz, T. Darden, H. Lee and L. Pedersen, J. Chem. Phys., 1995, 103, 8577-8593.

29 B. Hess, H. Bekker, H. Berendsen and J. Fraaije, J. Comput. Chem., 1997, 18, 1463-1472.

30 H. Berendsen, J. Postma, W. van Gunsteren, A. DiNola and J. Haak, J. Chem. Phys., 1984, 81, 3684-3690.

31 J. Ryckaert and A. Bellemans, Faraday Discuss. Chem. Soc., 1978, 95-106.

32 V. Bahamonde-Padilla, J. Espinoza, B. Weiss-Lopez, J. L. Cascales, R. Montecinos and R. Araya-Maturana, J. Chem. Phys., 2013, 139, 14703-14711.

33 N. Schmid, A. Eichenberger, M. Winger, A. Mark and W. van Gunsteren, Eur. Biophys. J., 2011, 7, 843-856.

34 J. Pople and G. Segal, J. Chem. Phys., 1966, 44, 3289.

35 B. Jonsson, O. Edholm and O. Teleman, J. Chem. Phys., 1986, 85, 2259-2271.

36 E. Egberts, S. Marrink and H. Berendsen, Eur. Biophys. J., 1994, 22, 423-436.

37 P. Ahlstrom and H. Berendsen, J. Phys. Chem., 1993, 97, 13691-13702. 
38 J. J. López Cascales, J. García de la Torre, S. Marrink and H. Berendsen, J. Chem. Phys., 1996, 104, 2713-2720.

39 P. David, W. van Gunsteren and A. Mark, J. Comput. Chem., 2010, 31, 1117-1125.

40 T. Heinz, W. van Gunsteren and P. Hünenberger, J. Chem. Phys., 2001, 115, 1125-1136.

41 H. Berendsen, J. Postma, W. van Gunsteren and J. Hermans, Intermolecular Forces, D. Reidel Publishing Company, 1981.

42 G. Raffard, S. Steinbruckner, A. Arnold, J. H. Davis and E. J. Dufourc, Langmuir, 2000, 16, 7655-7662.

43 L. S. Vermeer, B. L. de Groot, V. Réat, A. Milon and J. Czaplicki, Eur. Biophys. J., 2007, 36, 919-931.

44 M. Bloom, J. H. Davis and A. L. Mackay, Chem. Phys. Lett., 1981, 80, 198-202.

45 Biological Membranes. A Molecular Perspective from Computation and Experiment, ed. K. Merz and B. Roux, Birkhäuser, Boston, 1996.

46 M. Brown, R. Thurmond, S. Dodd, D. Otten and K. Beyer, J. Am. Chem. Soc., 2002, 124, 8471-8484.

47 H. Stegemeyer and H. Behret, Liquid Crystals, Steinkopff, 1994.

48 A. A. de Melo Filho, A. Laverde and F. Y. Fujiwara, Langmuir, 2003, 19, 1127-1132.

49 M. Orsi, D. Haubertin, W. Sanderson and J. Essex, J. Phys. Chem. B, 2008, 112, 802-815.
50 N. Chu, N. Kucerka, Y. Liu, S. Tristram-Nagle and J. Nagle, Phys. Rev. E: Stat., Nonlinear, Soft Matter Phys., 2005, 71, 71.041904.

51 W. Rawicz, K. Olbrich, T. McIntosh, D. Needham and E. Evans, Biophys. J., 2000, 79, 328-339.

52 H. Petrache, S. Tristram-Nagle, K. Gawrisch, D. Harries, V. Parseguian and J. Nagle, Biophys. J., 2004, 86, 1574-1586.

53 J. Pan, S. Tristram-Nagle, N. Kucerka and J. Nagle, Biophys. J., 2008, 94, 117-124.

54 H. Seto, N. Yamada, M. Nagao, M. Hishida and T. Takeda, Eur. Phys. J. E: Soft Matter Biol. Phys., 2008, 26, 217-223.

55 J. J. Lopez Cascales, S. D. Oliveira Costa, A. Garro and R. D. Enriz, RSC Adv., 2012, 2, 11743-11750.

56 M. Claessens, B. van Oort, F. Leermakers, F. Hoekstra and M. C. Stuart, Biophys. J., 2004, 87, 3882-3893.

$57 \mathrm{H}$. Elias, Macromolecules: structure and properties, Plenum Press, 1984, vol. 1, p. 7420.

58 S. Harding, Prog. Biophys. Mol. Biol., 1997, 68, 207-262.

59 R. Pamies, J. H. Cifre, M. L. Martínez and J. de la Torre, Colloid Polym. Sci., 2008, 286, 1223-1231.

60 J. García de la Torre, S. Navarro, M. L. Martínez and J. J. López Cascales, Biophys. J., 1994, 67, 530-531.

61 A. Seelig and J. Seelig, Biochemistry, 1974, 13, 4839-4845. 